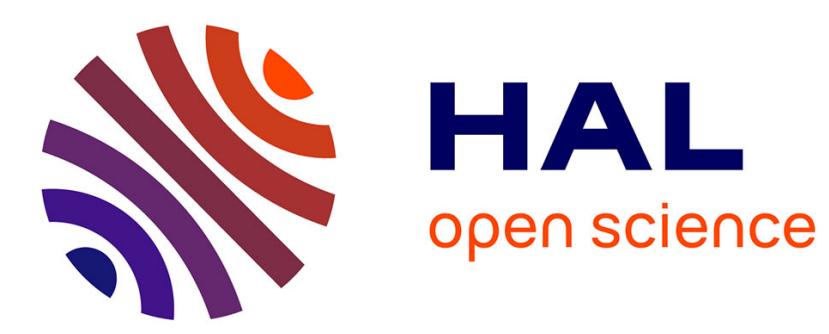

\title{
Anisotropic Turbulent Spectra in the Magnetosheath as Seen by the Cluster Spacecraft
}

\author{
F. Sahraoui, G. Belmont, L. Rezeau, N. Cornilleau-Wehrlin, Jean-Louis
}

Pinçon, A. Balogh

\section{To cite this version:}

F. Sahraoui, G. Belmont, L. Rezeau, N. Cornilleau-Wehrlin, Jean-Louis Pinçon, et al.. Anisotropic Turbulent Spectra in the Magnetosheath as Seen by the Cluster Spacecraft. Physical Review Letters, 2006, 96 (7), pp.075002. 10.1103/PhysRevLett.96.075002 . hal-00153449

HAL Id: hal-00153449

https://hal.science/hal-00153449

Submitted on 30 Apr 2020

HAL is a multi-disciplinary open access archive for the deposit and dissemination of scientific research documents, whether they are published or not. The documents may come from teaching and research institutions in France or abroad, or from public or private research centers.
L'archive ouverte pluridisciplinaire HAL, est destinée au dépôt et à la diffusion de documents scientifiques de niveau recherche, publiés ou non, émanant des établissements d'enseignement et de recherche français ou étrangers, des laboratoires publics ou privés. 


\title{
Anisotropic Turbulent Spectra in the Terrestrial Magnetosheath as Seen by the Cluster Spacecraft
}

\author{
F. Sahraoui, G. Belmont, L. Rezeau, and N. Cornilleau-Wehrlin \\ Centre d'Etude des Environnements Terrestre et Planétaires, IPSL, 10/12 avenue de l'Europe, 78140, Vélizy, France
}

\author{
J. L. Pinçon
}

Laboratoire de Physique et de Chimie de l'Environnement, 3A avenue de la Recherche Scientifique, 45071, Orléans, Cedex 2, France

A. Balogh

Space and Atmospheric Group, Blackett laboratory, Imperial College, Prince Consort Road, London, United Kingdom

(Received 27 June 2005; published 22 February 2006)

\begin{abstract}
Here we report the first three-dimensional spatial spectrum of the low frequency magnetic turbulence obtained from the four Cluster spacecraft in the terrestrial magnetosheath close to the magnetopause. We show that the turbulence is compressible and dominated by mirror structures, its energy is injected at a large scale $k \rho \sim 0.3(l \sim 2000 \mathrm{~km})$ via a mirror instability well predicted by linear theory, and cascades nonlinearly and unexpectedly up to $k \rho \sim 3.5(l \sim 150 \mathrm{~km})$, revealing a new power law in the inertial range not predicted by any turbulence theory, and its strong anisotropy is controlled by the static magnetic field and the magnetopause normal.
\end{abstract}

DOI: 10.1103/PhysRevLett.96.075002

Magnetic turbulence is ubiquitous in space and astrophysical plasmas (terrestrial magnetosphere, solar wind, interstellar medium, ...) [1-5]. Extensive studies, theoretical and experimental, have been devoted to understanding its role in fundamental processes occurring in magnetized plasmas such as mass transport, energy dissipation, and magnetic reconnection [6-9]. However, the experimental properties of this turbulence, particularly its spatial spectra, have not been determined hitherto unambiguously [10] because of the absence of data measured simultaneously at different points in space. From data acquired by one single spacecraft, the variations that are intrinsically temporal cannot be distinguished from those due to the exploration of spatial structures; the 3D shapes of the spatial structures are a fortiori inaccessible. Similarly, when analyzing turbulence in Fourier space, the spacecraft frequencies $f_{\mathrm{sc}}$ inextricably merge the frequency $f_{v}$ in the flow frame, which characterizes intrinsic temporal variations, and the Doppler shift $f_{D}=\mathbf{k} \cdot \mathbf{v} / 2 \pi$, which derives from spatial variations, the three frequencies being related by $f_{\mathrm{sc}}=f_{v}+f_{D}$ (the subscripts "sc" and " $v$ " denote, respectively, the spacecraft and flow reference frames). When the Taylor hypothesis is valid $\left(f_{v} \ll f_{D}\right)$, or when using two spacecraft data, it is possible to remove only partially this spatiotemporal ambiguity, i.e., only along the flow direction or along the axis separating the two satellites (see $[1,10]$ for a complete review). On the contrary, when using four spacecraft, the full 3D spatial spectrum can be obtained with much less restricting hypotheses as we will show here (see also [10,11]).

The data used here were measured by the four Cluster satellites [12] on 18 February 2002 about 5:34 UT in the magnetosheath, i.e., in the part of solar wind that is down-
PACS numbers: 94.30.Va, 52.35.Mw, 52.35.Py, 52.35.Ra

stream of the terrestrial bow shock and just in front of the outer boundary of the magnetosphere, the magnetopause. The spacecraft were at about $1 R_{E} \sim 6400 \mathrm{~km}$ from the magnetopause, and separated from each other by about $100 \mathrm{~km}$. Figure 1 shows the magnetic fluctuations measured by the flux gate magnetometer (FGM), sampled at $23 \mathrm{~Hz}$ [13]. FGM provides also the continuous components $\mathbf{B}_{\mathbf{0}}$ of the magnetic field, allowing us to define the magnetic field aligned reference frame $\left(z\right.$ axis aligned with $\left.\mathbf{B}_{\mathbf{0}}\right)$ in

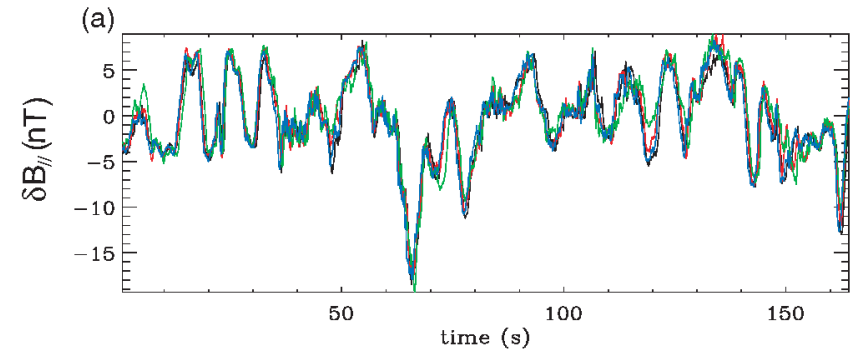

(b)

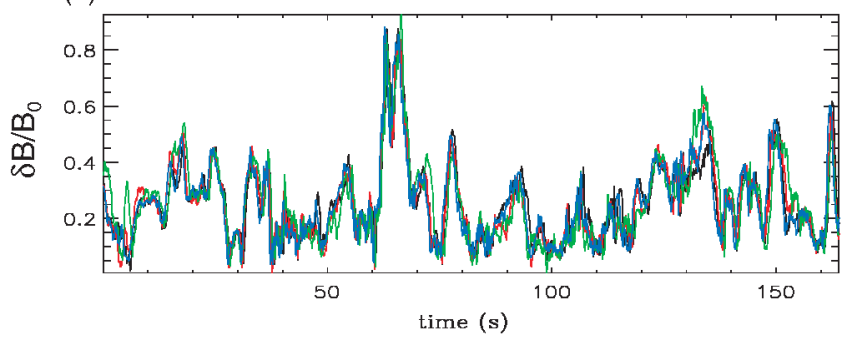

FIG. 1 (color). The magnetic field fluctuations measured by FGM in the magnetosheath. (a) The parallel component (to $\mathbf{B}_{\mathbf{0}}$ ) of the magnetic fluctuations. (b) The magnitude of the whole fluctuations normalized to the mean static field $B_{0}$. Four colors are used, one for each satellite. 
which these data are represented. We notice the strong quasiregular oscillation (of a period $\sim 10 \mathrm{~s}$ ) on the parallel component of these fluctuations [Fig. 1(a)], which is likely to be the signature of strong compressible waves. Note also the high level of these fluctuations $(\sim 40 \%)$ compared to the local static magnetic field [Fig. 1(b)] (a complete presentation of the data is given in $[10,14])$.

For studying turbulence, one has to investigate the power spectra which provide information on the scales and processes by which the energy is injected, transferred, and dissipated in the system. Figure 2 shows the low frequency part of the corresponding temporal spectra. The fastFourier-transform (FFT)s are calculated using a sliding window of a length $\sim 45 \mathrm{~s}$ (1024 points), over the whole interval $\sim 164 \mathrm{~s}$, multiplied by a $\cos ^{3}$ window function to avoid the boundary effect. The final spectrum results from an average over 30 subintervals (i.e., FFTs). The limitation to the range $(0-2) \mathrm{Hz}$ is due to the Cluster separation $(\sim 100 \mathrm{~km})$ and the given characteristic velocity $(\sim 200 \mathrm{~km} / \mathrm{s})$ to avoid the spatial aliasing effect in spectral analysis of turbulence [10]. The obtained spectrum (Fig. 2) has a maximum at the frequency $f_{\mathrm{sc}}=0.11 \mathrm{~Hz}$ and obeys approximately a power law $f^{-7 / 3}$ in the inertial range.

To investigate the physics involved in these spectra, one has to calculate how the energy is spatially distributed for each temporal frequency of the previous spectra, i.e., correct from the Doppler effect, and determine the corresponding scale lengths. The flow velocity used here $(v=220 \mathrm{~km} / \mathrm{s})$ is measured by the cyclotron ion spectrometer onboard Cluster [15]. For this purpose we have used the $k$-filtering technique. It is a generalized minimum variance analysis combining the 12 components of the magnetic field fluctuations (three per satellite), and allowing us to obtain the best estimate of the spectral energy density in the full $4 \mathrm{D}$ space $\left(f_{\mathrm{sc}}, \mathbf{k}\right)$, under the assumptions of weak time stationarity and space homogeneity of the time series [11]. This spectral energy is estimated by using nonlinear filters, in which it is possible to include any available constraint on the data. Here the physical condi-

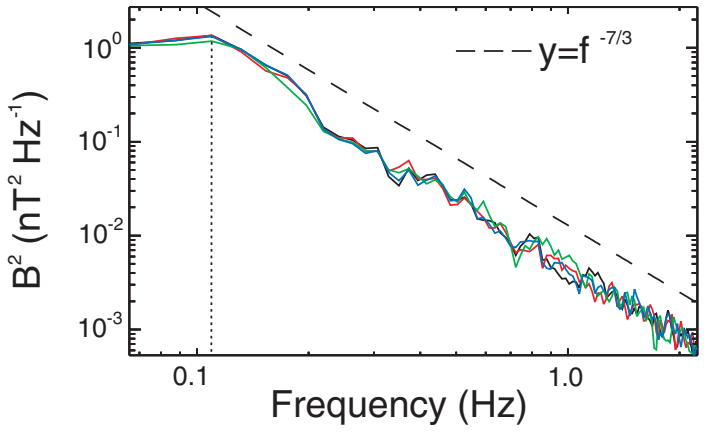

FIG. 2 (color). The temporal FFT spectra of the magnetic fluctuations, compared to the curve $f^{-7 / 3}$ (dashed line). The vertical dotted line is the frequency corresponding to the most intense observed wave. tion $\nabla \cdot \delta \mathbf{B} \equiv \mathbf{k} \cdot \delta \mathbf{B}=0$ has been considered while processing the $k$ filtering (for a detailed description see $[10,14,16])$. At the frequency $f_{\text {sc }}=0.11 \mathrm{~Hz}$, the energy density in $\mathbf{k}$ space is shown to have a main maximum [Fig. 3(a)] for wave vector components corresponding to spatial sizes about 10000,3500 , and $1800 \mathrm{~km}$ in the three directions $\mathbf{B}_{\mathbf{0}}$, $\mathbf{n}$, and $\mathbf{v}$ (defined in the legend of Fig. 3). The frequency $f_{v}$ of this maximum in the flow frame can then be obtained by correcting from the Doppler shift: $f_{v}=f_{\mathrm{sc}}-\mathbf{k} \cdot \mathbf{v} / 2 \pi$. The obtained value, $f_{v} \sim 0$, shows that the "wave" is actually a stationary structure in the flow frame, the observed frequency $f_{\mathrm{sc}} \sim 0.3 f_{\mathrm{cp}}\left(f_{\mathrm{cp}}=\right.$ $0.33 \mathrm{~Hz}$ is the proton gyrofrequency) being entirely due to Doppler shift.

To identify the nature of this structure, we have compared its experimental characteristics to the prediction of the kinetic linear theory of the "mirror" instability, which is known to grow in the magnetosheath plasma because of the proton temperature anisotropy $T_{\perp}>T_{\|}$when the criterion $\frac{T_{\perp}}{T_{\|}}-1 \geq \frac{1}{\beta_{\perp}}$ is satisfied $[17,18]$. Here, the measured plasma parameters are found to be very close to the theoretical instability threshold: the anisotropy parameter $A_{p}=$ $\frac{T_{\perp}}{T_{\|}}-1 \sim 0.28$ and the plasma beta $\beta \sim 4$. Moreover, Fig. 4
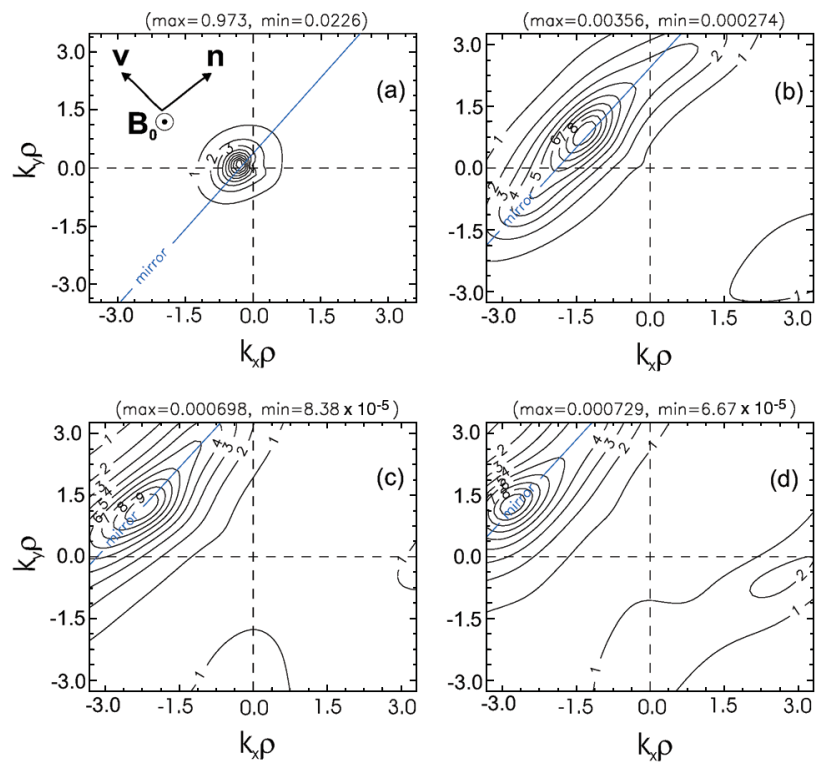

FIG. 3 (color). Stationary mirror structures identified by the $k$-filtering technique. The black curves are the isocontours of the energy density in the $\left(k_{x}, k_{y}\right)$ plane for four given frequencies: $f_{\text {sc }} \sim 0.3 f_{\text {cp }}(\mathrm{a}), 2 f_{\text {cp }}(\mathrm{b}), 3.5 f_{\text {cp }}(\mathrm{c}), 4 f_{\text {cp }}(\mathrm{d})$. The $k$ values are normalized to the inverse proton Larmor radius $(\rho \sim 75 \mathrm{~km})$. The levels 1-9 denote the lowest to highest energy values. These maxima lie almost at a constant $k_{\|},\left(\mathbf{k}, \mathbf{B}_{\mathbf{0}}\right) \sim 100^{\circ}$. The blue line is the Doppler shift $f_{\text {sc }}=\mathbf{k} \cdot \mathbf{v} / 2 \pi$. The arrows $\mathbf{B}_{\mathbf{0}}, \mathbf{n}$, and $\mathbf{v}$ are, respectively, the directions along the local static magnetic field, the normal to the magnetopause and the flow velocity, which form here a quasiorthogonal trihedron: $(\mathbf{v}, \mathbf{n}) \sim 104^{\circ}$, $\left(\mathbf{n}, \mathbf{B}_{\mathbf{0}}\right) \sim 81^{\circ}$, and $\left(\mathbf{B}_{\mathbf{0}}, \mathbf{v}\right) \sim 110^{\circ}$. 


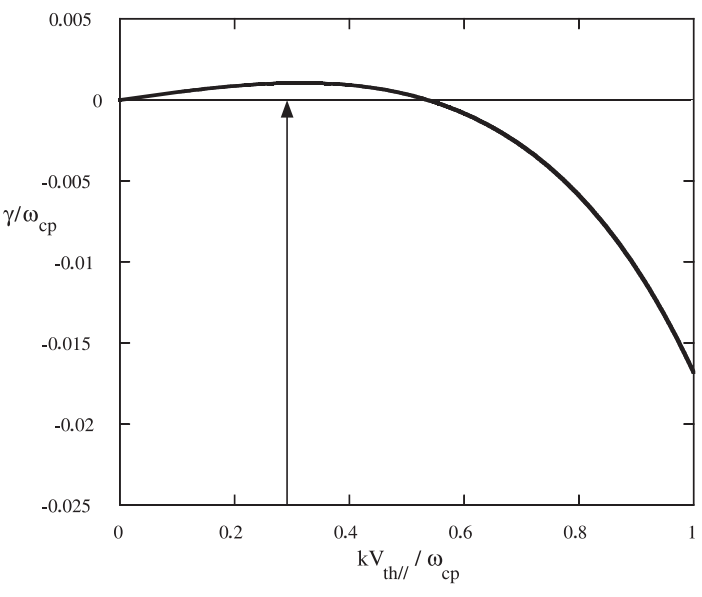

FIG. 4. The theoretical linear growth rate of the mirror instability calculated from the WHAMP program [23] for the angle $\left(\mathbf{k}, \mathbf{B}_{\mathbf{0}}\right) \sim 100^{\circ}$ and using the measured parameters of the plasma. $v_{\mathrm{th}_{\|}} \sim 154 \mathrm{~km} / \mathrm{s}$ is the proton parallel thermal velocity and $\omega_{\mathrm{cp}} \sim 2.07 \mathrm{rd} / \mathrm{s}$ is the proton cyclotron pulsation. The vertical arrow shows the location of the wave vector $(k \rho \sim$ $0.3)$ identified at $f_{\mathrm{sc}} \sim 0.11 \mathrm{~Hz}$. The scales $k \rho>0.5(\gamma<0)$ are strongly damped.

shows that the observed spatial scales lie accurately on the maximum of the linear growth rate of the mirror instability, proving thus that the linear mirror instability is responsible for injecting the energy of the spectrum of Fig. 2 at large scale. Similar mirror structures have been observed near other magnetized planets (Jupiter, Saturn) and comets $[19,20]$.

To evidence the turbulent cascade, we have performed the same study on all the higher spacecraft frequencies and shown that all the accessible scales are also dominated by similar stationary structures, with spatial scales decreasing with increasing frequencies [Figs. 3(b)-3(d)]. These small structures $(k \rho \geq 1)$ had not been observed before. Although they all prove to be stationary in the plasma frame $\left(f_{v} \sim 0\right)$, these fluctuations are observable at quite high frequencies in the spacecraft frame (up to $f_{\mathrm{sc}} \sim 5 f_{\mathrm{cp}}$ ). This result strikingly emphasizes the importance of the Doppler shift in the measured temporal spectra.

Figure 5 shows the spatial distribution of the energy density of each identified mirror structure along the principal axes $\mathbf{B}_{\mathbf{0}}, \mathbf{n}$, and $\mathbf{v}$. We observe that the energy decreases differently along these directions: very rapidly along $k_{\|}$indicating that there is no energy transfer parallel to $\mathbf{B}_{\mathbf{0}}$; fairly rapidly along the magnetopause normal with some spread without apparent order; more slowly and more regularly along the flow direction, leading to the formation of energetic small scales. These results provide a picture much more complete than ever of how the energy of magnetic turbulence is spatially spread near the magnetopause boundary. The largest mirror structure [Fig. 3(a)] can thus be viewed as a pumping source of energy, which cascades more preferentially along the flow direction, cre-
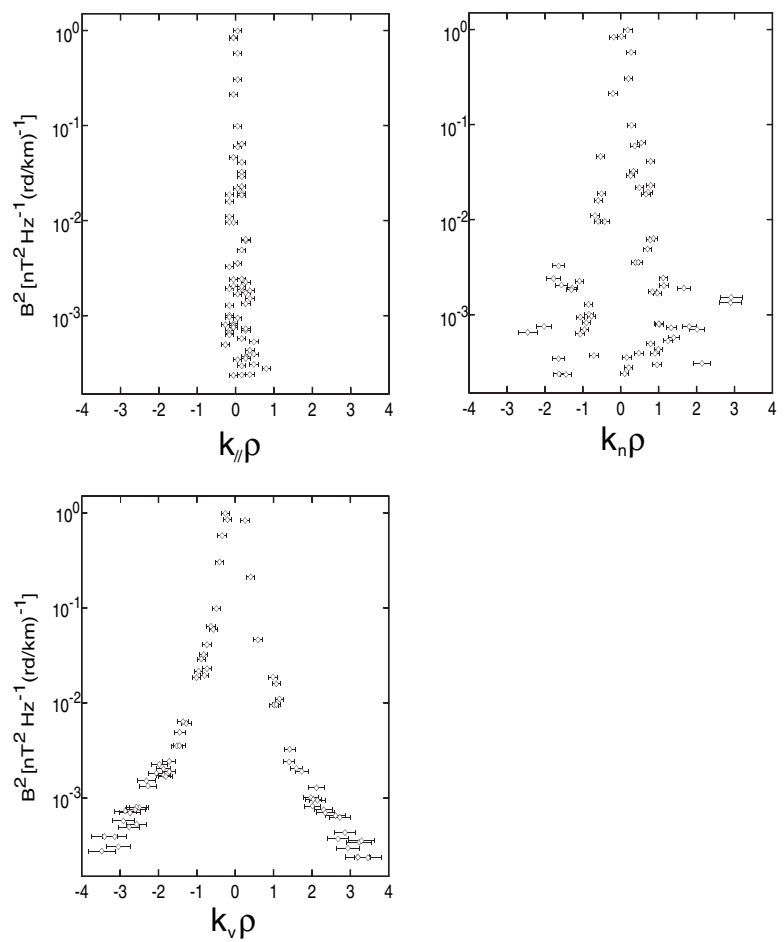

FIG. 5. A log-linear distribution of the energy density of the mirror structures identified for all the frequencies $f_{\text {sc }}$ of Fig. 2 as a function of their wave vector components (normalized to the inverse proton Larmor radius $\rho \sim 75 \mathrm{~km}$ ). The labels $\|, \mathbf{n}$, and $\mathbf{v}$ denote, respectively, the directions along $\mathbf{B}_{\mathbf{0}}$, the magnetopause normal and the flow. The horizontal lines are the error bars.

ating energetic small scales (up to $k_{v} \rho \sim 3.5$ ). As all the scales $k_{v} \rho \geq 1$ should have been strongly damped according to the kinetic linear theory of the mirror mode (Fig. 4), their observation [Figs. 3(b)-3(d)] suggests therefore that nonlinear effects are at work to overcome the linear damping. The error bars provided in Figs. 5 and 6 are related to two major sources of error: the uncertainty on the spacecraft positions $(\delta r / r<5 \%$ yielding $\delta k / k \sim 8 \%)$ [10], and the resolution in $\mathbf{k}$ space $(\delta k \sim 0.0014 \mathrm{rd} / \mathrm{km})$, limited by the stationarity and homogeneity of the signal $[10,16]$. The former dominates at large $k$ values, whereas the latter prevails at small $k$. Here we have considered an overestimated error of $\delta k / k=10 \%$ (a more complete description of these technical details will be given elsewhere).

The crucial point for turbulence theories is to determine the scaling law, which describes how the energy is transferred across scales over the inertial range. The obtained spectrum (Fig. 6) shows that most of the energy of the mirror structures cascades along the flow direction with a power law close to $k_{v}^{-8 / 3}$. This $1 \mathrm{D}$ spatial spectrum certainly dominates the temporal one in the spacecraft frame explaining the close values of the two indexes $7 / 3$ and $8 / 3$ (Fig. 6). We notice that the new power law $k_{v}^{-8 / 3}$ is steeper than all those predicted by the existing magnetohydrodynamiclike theories [4,5], which are based on oversimplify- 


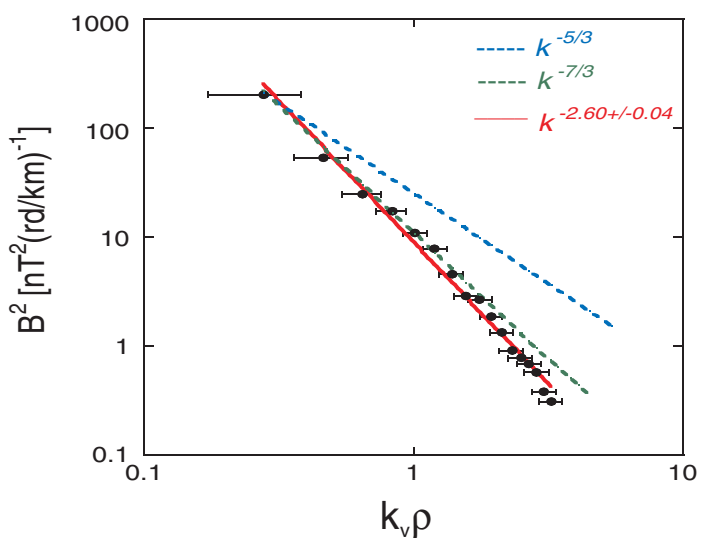

FIG. 6 (color). A log-log plot of the "reduced spectrum" of the magnetic energy as a function of the wave vector component $k_{v}$ along the flow $\mathbf{v}$ direction (normalized to the inverse proton Larmor radius). It is obtained after a double integration: a temporal one over all the spacecraft frequencies of Fig. 2, providing the 3D spatial spectrum; a spatial one over the $\mathbf{B}_{\mathbf{0}}$ and $\mathbf{n}$ directions. The red line is a direct fit revealing a power law $k_{v}^{-2.6}$. Two other power laws are plotted for comparison: $k^{-7 / 3}$ (green) and $k^{-5 / 3}$ (blue). The upper limit $k_{v} \rho \sim 3.5\left(L_{v} \sim\right.$ $150 \mathrm{~km})$ is the smallest scale that can be measured with the $100 \mathrm{~km}$ Cluster separation.

ing assumptions like isotropy of incompressibility. It is worth noticing also that this spectrum does not break down below the ion scale $\left(k_{v} \rho \geq 1\right)$ as it was usually thought concerning the physics of the mirror mode [17].

The direct consequence of the above results concerns the modeling of turbulence. In the absence of a satisfactory theory to explain the generation of the observed small scales [17], we present the following scenario to explain, phenomenologically, the observed cascade: The anisotropic behavior (Fig. 5) suggests viewing the magnetic field $\mathbf{B}_{\mathbf{0}}$ and the magnetopause normal $\mathbf{n}$ as external constraints preventing the turbulence to develop "freely." The only direction "let free" from any constraint is thereby along v, allowing a 1D "fully developed turbulence" to settle only in this direction. A consistent theory should therefore consider the three ingredients evidenced here: the kinetic nature of the energy injection, the cascade scenario similar to those used in fluid turbulence [4,5], and the role of large scale inhomogeneity due to the magnetopause boundary. Using improved fluid numerical codes that catch the necessary kinetic effects [21], this new approach of turbulence should be feasible with the actual computing means. A further possible application of the present results concerns the reconnection problem. Indeed, the new scaling law presented here could be used as a new input for the turbulent reconnection models [6,7], which aim to explain fast reconnection in astrophysical plasmas by considering the large to small scale energy transfers. It can also be considered as an external constraint in numerical models devoted to study reconnection driving problems [22].

F. Sahraoui was funded by the Centre National d'Etudes Spatiales. The authors would like to thank J. M. Bosqued for providing the CIS data used in this work to compute the physical parameters of the plasma.

[1] C.P. Tu and E. Marsch, MHD Structures, Waves and Turbulence in the Solar Wind: Observations and Theories (Kluwer Academic, Belgium, 1997).

[2] W. H. Matthaeus and M. L. Goldstein, J. Geophys. Res. 87, 6011 (1982).

[3] M. L. Goldstein and D. A. Roberts, Phys. Plasmas 6, 4154 (1999).

[4] S. Galtier et al., J. Plasma Phys. 63, 447 (2000).

[5] D. Biskamp et al., Phys. Plasmas 6, 751 (1999).

[6] A. Lazarian, astro-ph/0505574.

[7] E. J. Kim and P. H. Diamond, Astrophys. J. 556, 1052 (2001).

[8] S. D. Bale et al., Phys. Rev. Lett. 94, 215002 (2005).

[9] G. Belmont and L. Rezeau, J. Geophys. Res. 106, 10751 (2001).

[10] F. Sahraoui et al., J. Geophys. Res. 108, 1335 (2003).

[11] J.L. Pinçon and F. Lefeuvre, J. Geophys. Res. 96, 1789 (1991).

[12] C. P. Escoubet et al., The Cluster and Phoenix Missions (Kluwer Academic, Belgium, 1995).

[13] A. Balogh et al., Ann. Geophys. 19, 1207 (2001).

[14] F. Sahraoui et al., Ann. Geophys. 22, 2283 (2004).

[15] H. Rème et al., Ann. Geophys. 19, 1303 (2001).

[16] S. N. Walker et al., Ann. Geophys. 22, 3021 (2004).

[17] R. A. Treumann et al., Nonlin. Proc. Geophys. 11, 647 (2004).

[18] S. P. Gary et al., J. Geophys. Res. 20, 1767 (1993).

[19] M.B.B. Cattaneo, G.C. Basile, and J.D. Richardson, J. Geophys. Res. 103, 11961 (1998).

[20] K.H. Glassmeier et al., J. Geophys. Res. 98, 20955 (1993).

[21] T. Passot and P. L. Sulem, Phys. Plasmas 11, 5173 (2004).

[22] J. Birn et al., Geophys. Res. Lett. 32, L06 105 (2005).

[23] K. Ronmark, Report 179 of Kiruna Geophysical Institute (Kiruna Geophysical Institute, Sweden, 1982). 